\title{
Observations of Mars with ALMA: potential for future constraints of global circulation models
}

\author{
Maxwell C. Parks, ${ }^{a, b}, *$ Conor A. Nixon, ${ }^{b}$ Geronimo L. Villanueva ${ }^{b}$, \\ Michael D. Smith, ${ }^{b}$ Alain S. J. Khayat, ${ }^{b, c}$ Alexander E. Thelen $\odot,{ }^{b, d}$ \\ Eric Villard, ${ }^{e}$ Steven B. Charnley, ${ }^{b}$ and Patrick G. J. Irwin ${ }^{f}{ }^{\prime}$ \\ ${ }^{a}$ University of Maryland, Center for Research and Exploration in Space Science \\ and Technology II, Baltimore, Maryland, United States \\ bNASA Goddard Space Flight Center, Greenbelt, Maryland, United States \\ 'University of Maryland, Center for Research and Exploration in Space Science \\ and Technology II, College Park, Maryland, United States \\ ${ }^{\mathrm{d}}$ Universities Space Research Association, Washington, D.C., United States \\ 'Joint ALMA Observatory/ESO, Santiago, Chile, United States \\ fOxford University, Oxford, United Kingdom
}

\begin{abstract}
Validation of global climate models (GCMs) for planets in our solar system requires observational data, but observations from the orbit of Mars and its surface are limited in number and are constrained by their orbit or landing site. Ground-based observations of Mars can help by providing data across the entire Martian hemisphere, yet historically, ground-based observations at submillimeter wavelengths have been limited to disk-average, or at best, a few resolution elements across Mars. We used Atacama Large Millimeter/submillimeter Array (ALMA) observations of Mars to determine the spatial distribution of carbon monoxide in the Martian atmosphere, which can be related to the atmospheric temperature. ALMA's comparably high spatial and spectral resolutions in the submillimeter wavelengths could allow the mapping of abundances and temperature profiles, and the comparison of these data to simulations generated by the Laboratoire de Météorologie Dynamique (LMD) Mars GCM. However, the long baselines associated with the high spatial resolution of ALMA introduced systematic errors that resulted in radiative transfer modeling degeneracies. We serve to provide insight to facilitate proposed ALMA observations of Mars in the future so that the systematic errors encountered within these observations might be avoided. (C) 2021 Society of Photo-Optical Instrumentation Engineers (SPIE) [DOI: 10.1117/1.JATIS.7.2.025001]
\end{abstract}

Keywords: Mars; inner solar system; interferometry; radio; atmosphere; carbon monoxide.

Paper 20150 received Oct. 5, 2020; accepted for publication Mar. 18, 2021; published online Apr. 21, 2021.

\section{Introduction}

With a chemical lifetime of 3 to 6 years and a dynamical mixing time of $\sim 10$ days, ${ }^{1,2}$ carbon monoxide $(\mathrm{CO})$ in the Martian atmosphere is expected to be uniformly vertically mixed in the lower atmosphere (below $50 \mathrm{~km}$ ) and stable across Martian day-length (sol) timescales. Observations from spacecraft and ground-based observatories constrained the global average Martian CO abundance to $\sim 800 \mathrm{ppm} .{ }^{3,4}$ However, seasonal variations due to condensation of $\mathrm{CO}_{2}$ at the poles can cause variation in the average column mixing ratio from $200 \mathrm{ppm}$ during summer at the poles, to as much as $1500 \mathrm{ppm}$ during winter at the pole. These winter increases arise from a rise in $\mathrm{CO}$ mixing ratio as $\mathrm{CO}_{2}$ freezes out, leaving the atmosphere with enhanced $\mathrm{CO}$ proportions. In-situ measurements of the $\mathrm{CO}$ distribution in the atmosphere have also taken place since the Viking Landers arrived in 1976.,

With assumptions about the spatial and temporal abundance of Martian CO, we can use CO emission and absorption lines to measure temperature profiles across Mars. ${ }^{2,6-8}$ However, the

*Address all correspondence to Maxwell C. Parks, Maxwell.c.parks@nasa.gov

$2329-4124 / 2021 / \$ 28.00$ (C) 2021 SPIE

J. Astron. Telesc. Instrum. Syst.

025001-1

Apr-Jun 2021 • Vol. 7(2) 
data from spacecraft observations are constrained by the specific orbits and landing sites of each mission. For example, the Phobos spacecraft's Infrared SpectroMeter instrument was limited in its investigations to latitudes between $30^{\circ} \mathrm{N}$ and $30^{\circ} \mathrm{S}$ (as seen here Ref. 9), and the retrievals at near-IR wavelengths from Compact Reconnaissance Imaging Spectrometer for Mars aboard the Mars Reconnaissance Orbiter require reflected sunlight and so are limited to a solar incidence angle of less than $\sim 80 \mathrm{deg}$, which prevents observations of the winter poles. ${ }^{3,4}$

Ground-based observations, however, can be used to capture global snapshots of Mars, providing simultaneous data for the visible hemisphere of the planet. Full-disk images can be used to validate model predictions against observations by providing a view of a full half of the atmosphere at once, rather than only the portion directly observable by the orbiters currently in operation. In the past, ground-based submillimeter data were limited by spatial resolution. At its most distant, the disk of Mars is just 3.5" across, and even at opposition, Mars only has an angular diameter of up to $\sim 25^{\prime \prime}$. In comparison, submillimeter observatories such as Institut de Radioastronomie millimétrique (IRAM) $30 \mathrm{~m}$ telescope or the James Clerk Maxwell Telescope (JCMT), observing at $337 \mathrm{GHz}$, have half-power beam widths (HPBW) of $\sim 7^{\prime \prime}$ and $\sim 14^{\prime \prime}$, respectively. Therefore, even during opposition, previous ground-based observations have been limited to two to three beam-widths across Mars; for $\sim 60 \%$ of the 26 -month long cycle between oppositions, neither observatory is capable of resolving Mars at $337 \mathrm{GHz}$. One can observe in shorter wavelengths to improve resolution somewhat, per the Rayleigh criterion, but we chose the 337-GHz window due to availability of comparable data in the Atacama Large Millimeter/ submillimeter Array (ALMA) archive and clear signal from the $\mathrm{C}^{17} \mathrm{O}$ transition. The relationship between observatory resolution and angular diameter of Mars is shown in Fig. 1. Modern interferometers such as the higher-resolution Sub-Millimeter Array (SMA) provide a maximum HPBW of $\sim 0.3^{\prime \prime}$ in its most extended configuration, which would allow for a maximum of $\sim 10$ beam-widths across Mars even when the planets are at their most distant and up to $\sim 90$ beam-widths across Mars at opposition. With even more extended submillimeter interferometers such as ALMA, we can observe Mars at a higher spatial resolution than was previously possible; depending on the configuration, ALMA can reach spatial resolutions of less than 0.01 " at $337 \mathrm{GHz}$. This increased resolution enables us to collect data at spatial scales relevant to global atmospheric patterns.

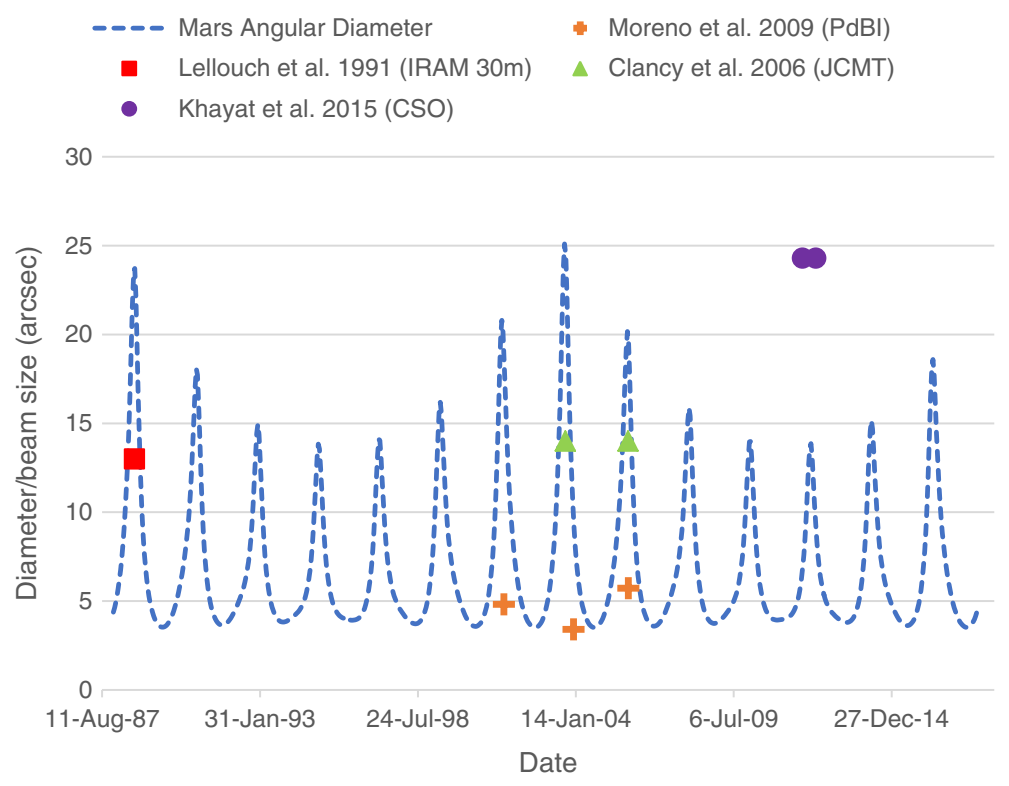

Fig. 1 A sample of beam sizes from submillimeter observations including single dish [IRAM $30 \mathrm{~m},{ }^{2}$ JCMT, ${ }^{10}$ Caltech Submillimeter Observatory ${ }^{6}$ (CSO)] and interferometric observations (Plateau de Bure Interferometer, ${ }^{11}$ or $\mathrm{PdBI}$ ), compared to the angular diameter of Mars when viewed from Earth. Even when observed at opposition, the Martian disk rarely spans more than $5 \times x$ the FWHM of the observing beam. 


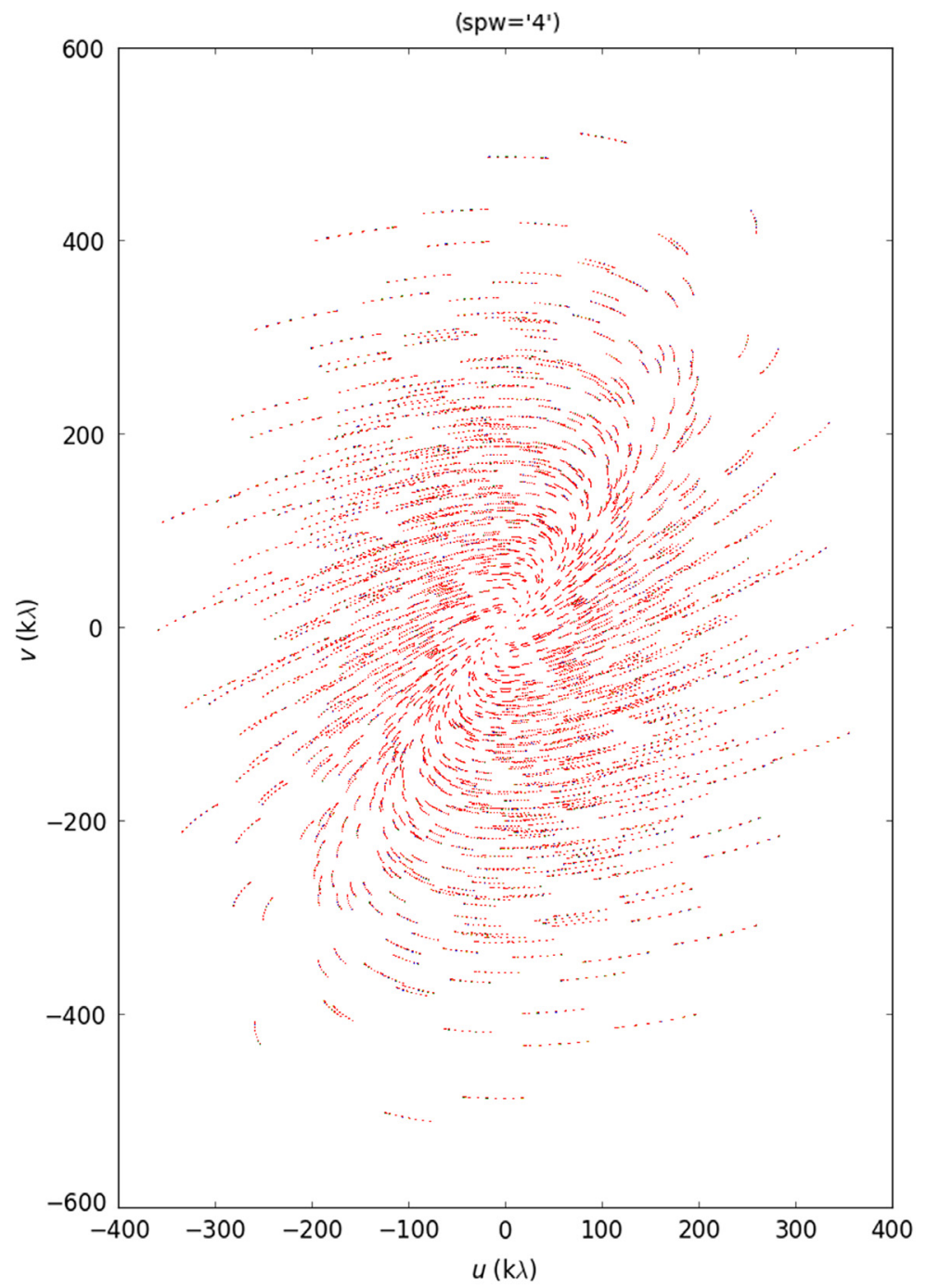

Fig. 2 Fourier transformed $u-v$ plane coverage for ALMA configuration C36-2 on March 13, 2016. This represents the sampling and shows the incomplete nature of any sampling of the $u-v$ plane.

This research aims to serve as a guideline to further develop the capacity for the latest generation of Earth-based submillimeter/radio interferometers to provide verification and validation pathways for global climate models (GCMs) of Mars. ALMA is particularly valuable in this capacity as its archive contains publicly available datasets of Mars from historic observations.

Here, we discuss the consequences of inadequately sampled short baselines for highresolution interferometric observations of Mars, which will inform future observations of Mars and enable better utilization of high-resolution interferometers in refining and informing GCMs for Mars. Section 2 will cover our observation parameters, and Sec. 3 will capture our data processing. Section 4 will discuss our efforts to fit the data with physically reasonable atmospheric models, and Sec. 5 establishes our conclusion that errors within our data stemming from inadequately sampled short baselines prevent us from accomplishing the high-fidelity atmospheric modeling we were attempting.

\section{ALMA Observation and Data Reduction}

Interferometric observations of Mars were made between UT 2016 March 13, 11:10 and UT 2016 March 13, 12:14, as the primary objective of ALMA project \#2015.1.01269.S 
(Three-dimensional mapping of the water cycle on Mars, PI: Villanueva). Solar longitude $\left(L_{s}\right)$ was $121.8 \mathrm{deg}$, for Mars year 33; at the time of observation, the angular diameter of Mars' disk was 9.8". The observations utilized ALMA Band 7 to cover a frequency range of 336.92 to $337.15 \mathrm{GHz}$. The spectral resolution of the observation achieved was $488.22 \mathrm{kHz}$. The integration time on source was $4020 \mathrm{~s}$, with thirty-eight $12-\mathrm{m}$ antennae providing baselines ranging from 15 to $460 \mathrm{~m}$. The ALMA array can also include a cluster of 7-m dishes called the Atacama Compact Array (ACA), to help capture signal from large angular scales, but it was not active during this observation. ALMA was in configuration C36-2 on the date of the observation leading to a maximum recoverable scale of $7.33^{\prime \prime}$ at $337 \mathrm{GHz}$. A spatial diagram of the antennas used can be found in Fig. 2.

Continuum-subtraction of the calibrated visibility data was performed using the uvcontsub task in the NRAO CASA software (version 4.2.1 ${ }^{12}$ ), and imaging was carried out using the clean task. Deconvolution of the point-spread function was performed for each spectral channel using the Högbom algorithm, with natural visibility weighting. The image pixel sizes were set to $0.1^{\prime \prime} \times 0.1^{\prime \prime}$. The resulting spatial resolution (FWHM of the Gaussian restoring beam at $337 \mathrm{GHz}$ ) was $0.716^{\prime \prime} \times 0.500^{\prime \prime}$. This resolution element corresponded to $496 \times 347 \mathrm{~km}$ at Mars's geocentric distance of $0.96 \mathrm{AU}$ at the time of observation (compared with Mars' $6779 \mathrm{~km}$ diameter). With around 18 beams across the disk of Mars, this was one of the highest resolution observations of Mars in the submillimeter wavelengths.

We created a map of the flux integrated over the width of the $\mathrm{C}^{17} \mathrm{O}(J=3$ to 2$)$ transition located at $337.06 \mathrm{GHz}$ (Fig. 3). Such a map represents the total integrated intensity of a spectral line, and with it, one can see the spatial distribution of $\mathrm{CO}$ emission at the edge of the Martian disk.

However, due to the configuration of ALMA at the time, the disk of Mars was beyond the maximum recoverable scale of the observatory. To understand maximum recoverable scale, one must understand that interferometry relies on taking the Fourier transform of the sky, which translates the image into a phase space called the $u-v$ plane. This is best described by the ALMA Technical Handbook: "an image is a "sum" (i.e., the Fourier transform) of the visibilities of where each visibility has an amplitude and phase representing the brightness and relative

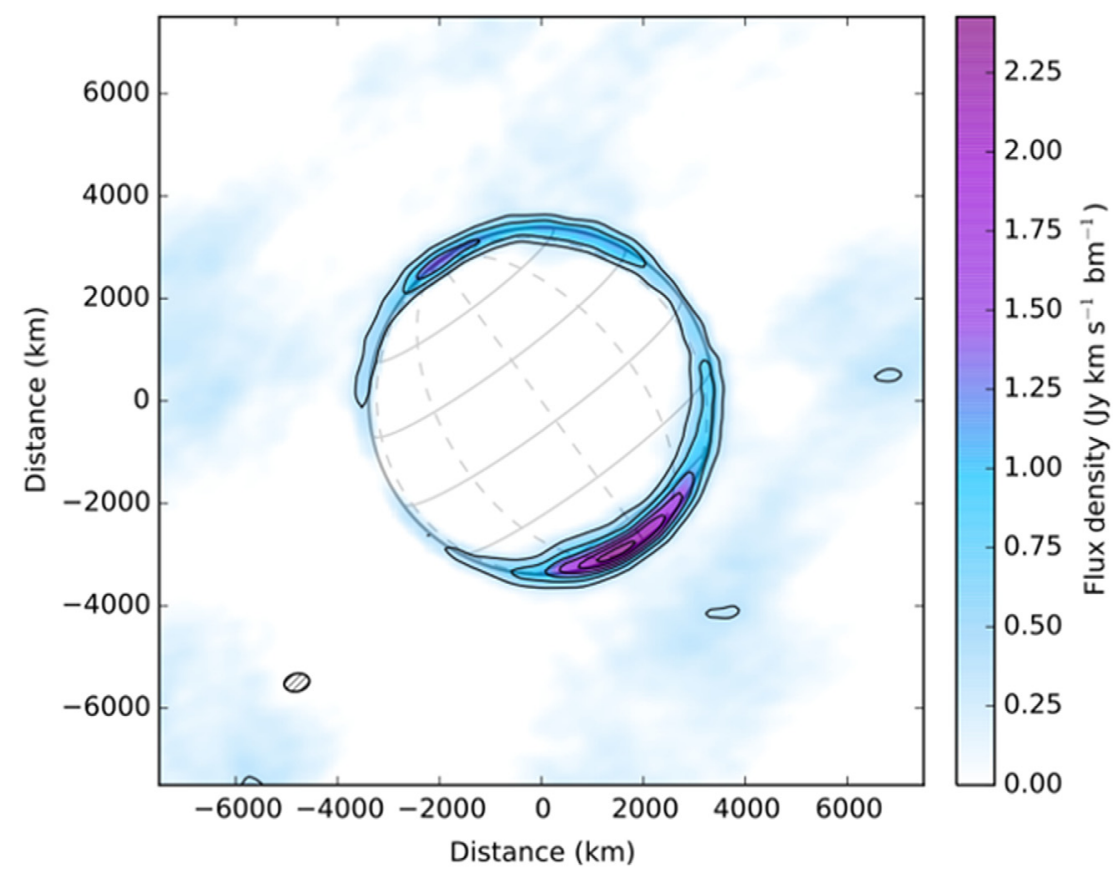

Fig. 3 Mars $\mathrm{C}^{17} \mathrm{O}(\mathrm{J}=3$ to 2$)$ transition integrated emission map from March 2016, following continuum-subtraction. Each contour level represents a $3-\sigma$ step in the total flux integrated over the emission line. The hatch-shaded ellipse in the bottom left represents the full-width half-maximum of the beam. 
position of emission on a specific angular scale." The specific angular scale is important. Because each specific angular scale is best captured by a specific corresponding baseline of the interferometer, the configuration of the telescope determines what is resolvable. If certain baselines are not represented in the configuration, the telescope cannot capture the full brightness of the angular scales corresponding to the missing baselines.

In our observation, long baselines between receivers, as shown in Fig. 2, resulted in higher spatial resolution, but without simultaneous observations at short baselines (i.e., with the ACA), the observations lacked adequate sampling of the $u-v$ plane to attain sensitivity to large-scale structures. Without this sampling, Mars was larger than the maximum recoverable scale, and there were inconsistent losses in continuum flux across the entire disk of Mars. These losses persisted even after calibration using the CASA calibration scripts provided by the Joint ALMA Observatory. The nonuniform pattern of flux loss is shown in Fig. 4, further illustrated by an example spectrum extracted from the south pole of Mars in Fig. 5.

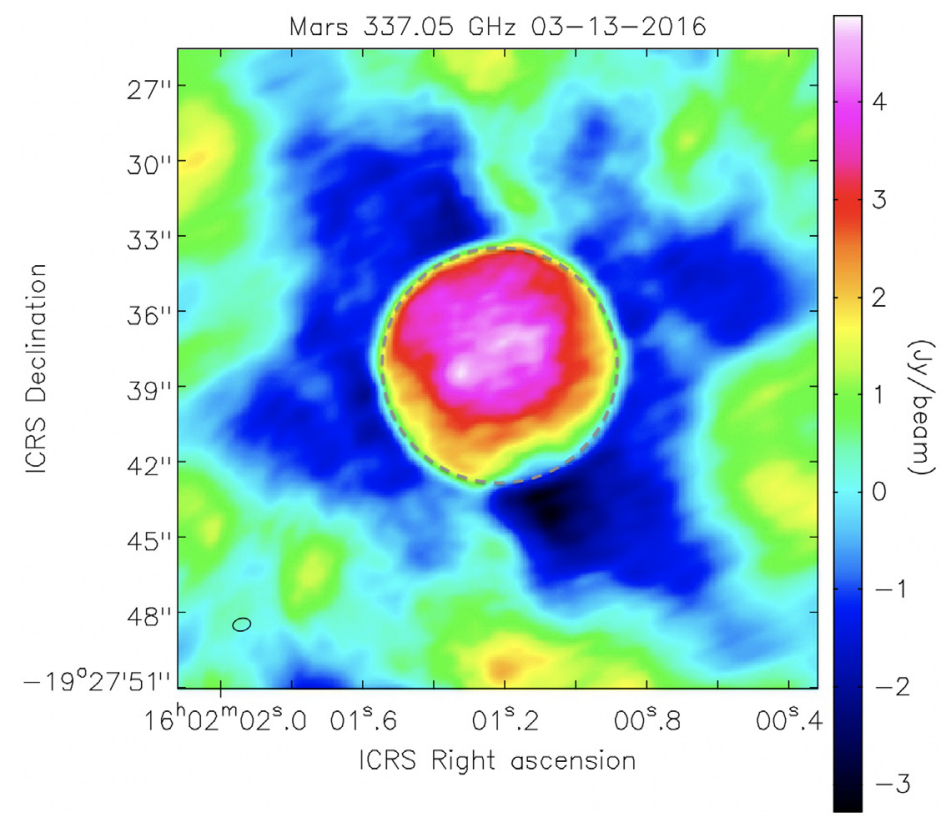

Fig. 4 A continuum image from the March 2016 ALMA observations of Mars at $337.05 \mathrm{GHz}$ with a dashed gray outline for Mars. Large-scale imaging artifacts are present due to the lack of short spacings in the ALMA array configuration (negative color bar regions). Note the negative Jy/beam overlapping the southern edge of Mars, shown in detail in Fig. 5.

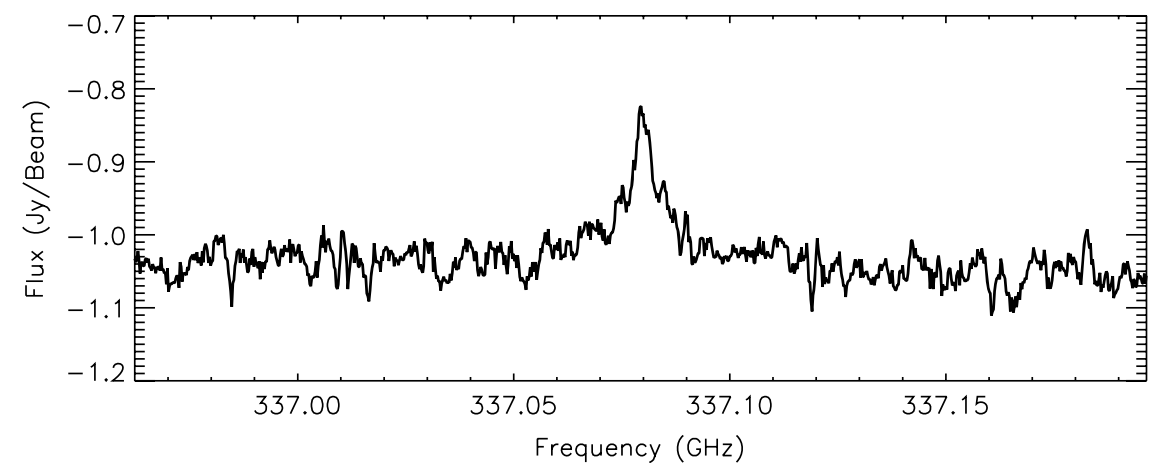

Fig. 5 A sample spectrum extracted from the southernmost point on the disk of Mars, in the midst of one of the flux loss areas. The calibrated flux of the $\mathrm{C}^{17} \mathrm{O}$ signal was negative, even for an emission line. This negative flux level meant any continuum data from this observation was not properly captured due the limited $u-v$ coverage of the interferometric observations. 


\section{Modeling}

The Laboratoire de Météorologie Dynamique (LMD), in Paris, France, has developed a GCM for Mars. ${ }^{13,14}$ Simulated data from this GCM can be found on the Mars Climate Database (MCD), which is managed by LMD. To demonstrate our ability to use ALMA archival data as a validating mechanism for GCMs, we acquired data on the state of the atmosphere (pressure, temperature, and $\mathrm{CO}$ abundance) at each latitude from the current version (5.3) of the MCD corresponding to the same date and time as our observations. We assumed terrestrial CO isotopic abundances for this investigation. Adapting these data to simulate what ALMA should perceive on the date of our observation, with appropriate adjustments to account for emission angle to the ALMA facility, provides simulated spectra with which to compare our observations.

We modeled the spectra in two parallel ways. We used the non-linear optimal estimator for multivariate spectral analysis (NEMESIS) radiative transfer retrieval code, ${ }^{15}$ which has previously been applied to analysis of Titan submillimeter spectra from ALMA ${ }^{16-18}$ and Herschel/SPIRE. ${ }^{19}$ We obtained spectral line widths from the HITRAN 2012 database ${ }^{20,21}$ as in Refs. 22 and 17 and subsequently adjusted the broadening coefficients to account for the $\mathrm{CO}_{2}$ dominated atmosphere of Mars as in Ref. 23. ${ }^{6}$ The spectral extraction was done by weighting the ALMA data using a version of the "beamrays_t17" code from Thelen and Molter (2017) (http://dx.doi.org/10.17632/szbcb44s43.1), modified for Mars, and followed the process laid out in Refs. 22 and 24. A diagram of emission angle weighting can be found in Fig. 6.

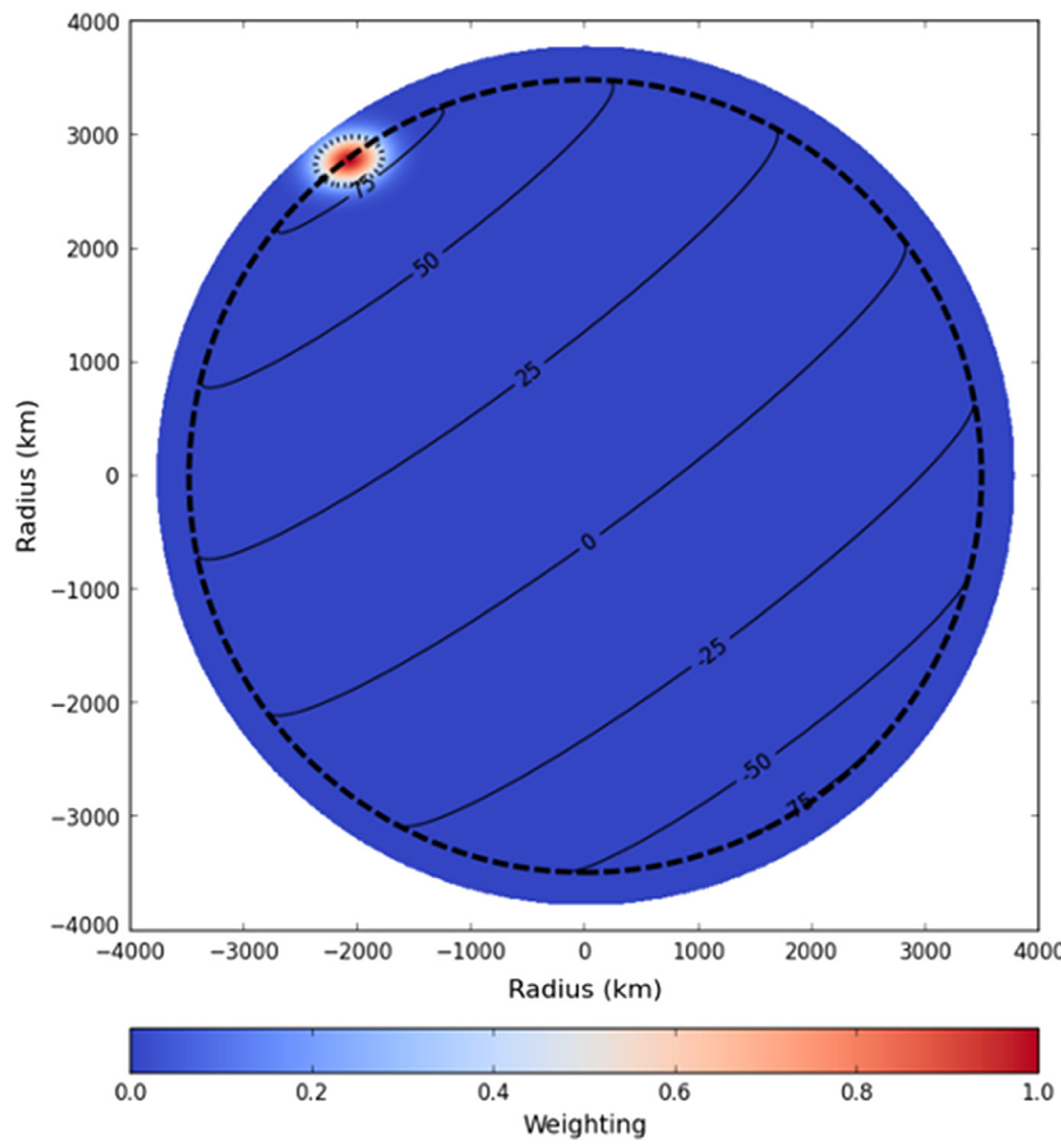

Fig. 6 Emission angle weighting for the $90^{\circ} \mathrm{N}$ latitude spectrum. Mars's solid disk is represented here as the dashed circle; the color scale represents weighting given to emission angles for radiative transfer modeling. The beam FWHM corresponding to each extracted spectrum is shown with a dotted ellipse. 


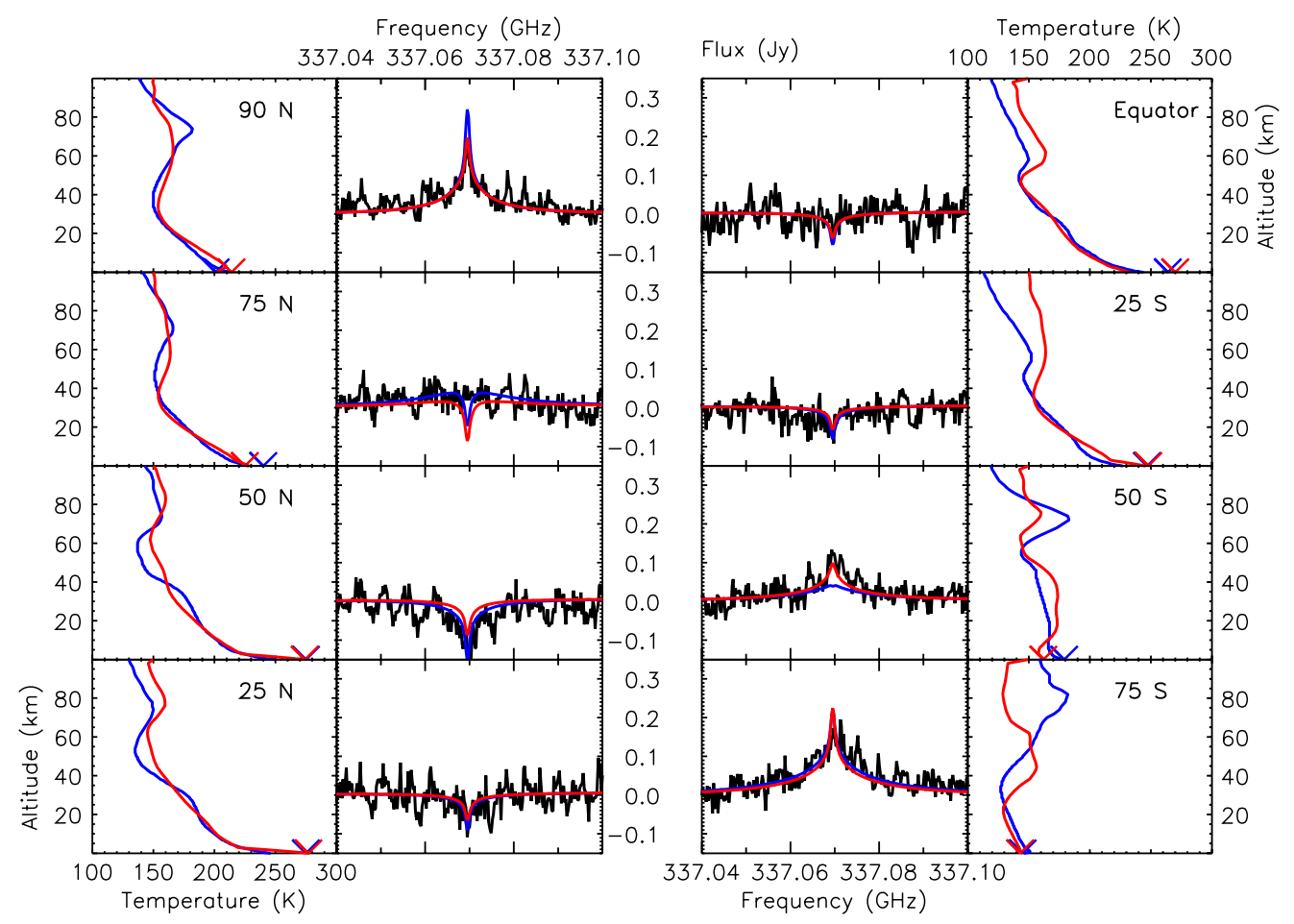

Fig. 7 A sample of vertical temperature profiles (first and last columns) and spectral lines (middle two columns) from a longitudinal swath at longitude $90^{\circ} \mathrm{E}$. The blue temperature profiles correspond to the GEM-Mars atmosphere models, and red denotes those from MCD. The respective spectral forward models from NEMESIS are compared to the ALMA data (black). Colored chevrons represent the surface temperatures from both GEM-Mars and MCD.

We intercompared the NEMESIS results with synthesized spectra from the Planetary Spectrum Generator (PSG). PSG is a publicly available ${ }^{25}$ radiative transfer suite $^{26}$ and served as a complementary tool to the NEMESIS forward models. PSG employs the latest HITRAN distribution (updated March 2020), with $\mathrm{CO}_{2}$ broadening coefficients for the rotational $\mathrm{CO}$ lines sampled in this study. In addition to corroborating our modeling using NEMESIS, PSG was also used to query temperature results from the Global Environmental Multiscale (GEM) Mars model, allowing further comparison of our computed spectra. GEM-Mars is a three-dimensional GCM for the atmosphere of Mars based on the standard weather forecast model of Environment Canada. ${ }^{27}$

Samples of the continuum subtracted ALMA Spectra are shown in Figs. 7 and 8, for a longitudinal swath along the $90^{\circ} \mathrm{E}$ Meridian and the $180^{\circ} \mathrm{E}$ Meridian, respectively. Forward models using both the MCD and GEM-Mars atmospheric models are plotted along with their respective temperature profiles.

\section{Results}

By comparing the absorption and emission lines at different latitudes (Figs. 9 and 10), we intended to gain insight into the similarities between the predicted state of the atmosphere and the observation. To account for the negative continuum discussed in Figs. 4 and 5, we subtracted the continuum average from the data sets for each location. This would allow one to compare line shapes directly, and let one add synthetic continuums to the emission lines for the purpose of retrieving atmospheric properties. Initial assessment of sampled regions from the disk showed some agreement, as shown in Figs. 7 and 9; predicted $\mathrm{CO}$ enhancement in the winter pole. However, the consistent offset between observation and model in Figs. 8 and 10 indicated that flux was lost not only in the continuum but also in limb emission. Systematic error in the limb emission lines would result in an analytical degeneracy: even without physically plausible continuum levels, one could simulate continuum flux to perform retrievals on the emission and 


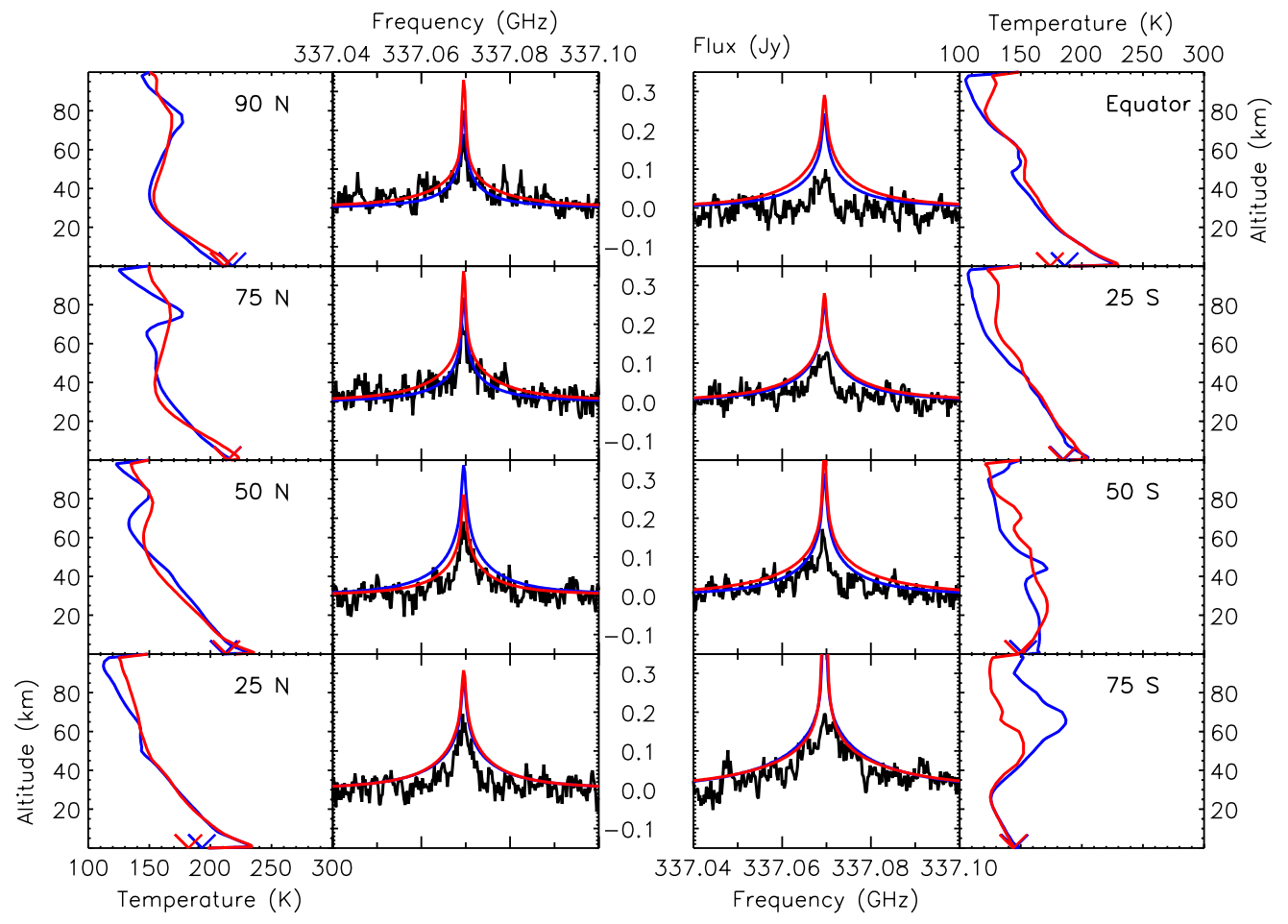

Fig. 8 To compare the modeled emission features at the limb, we plotted temperature profiles (first and last columns) and spectral lines (middle two columns) from a longitudinal swath at $180^{\circ} \mathrm{E}$, at the same latitudes as Fig. 7. As in Fig. 7, the blue line is GEM Mars and the red line is from MCD.

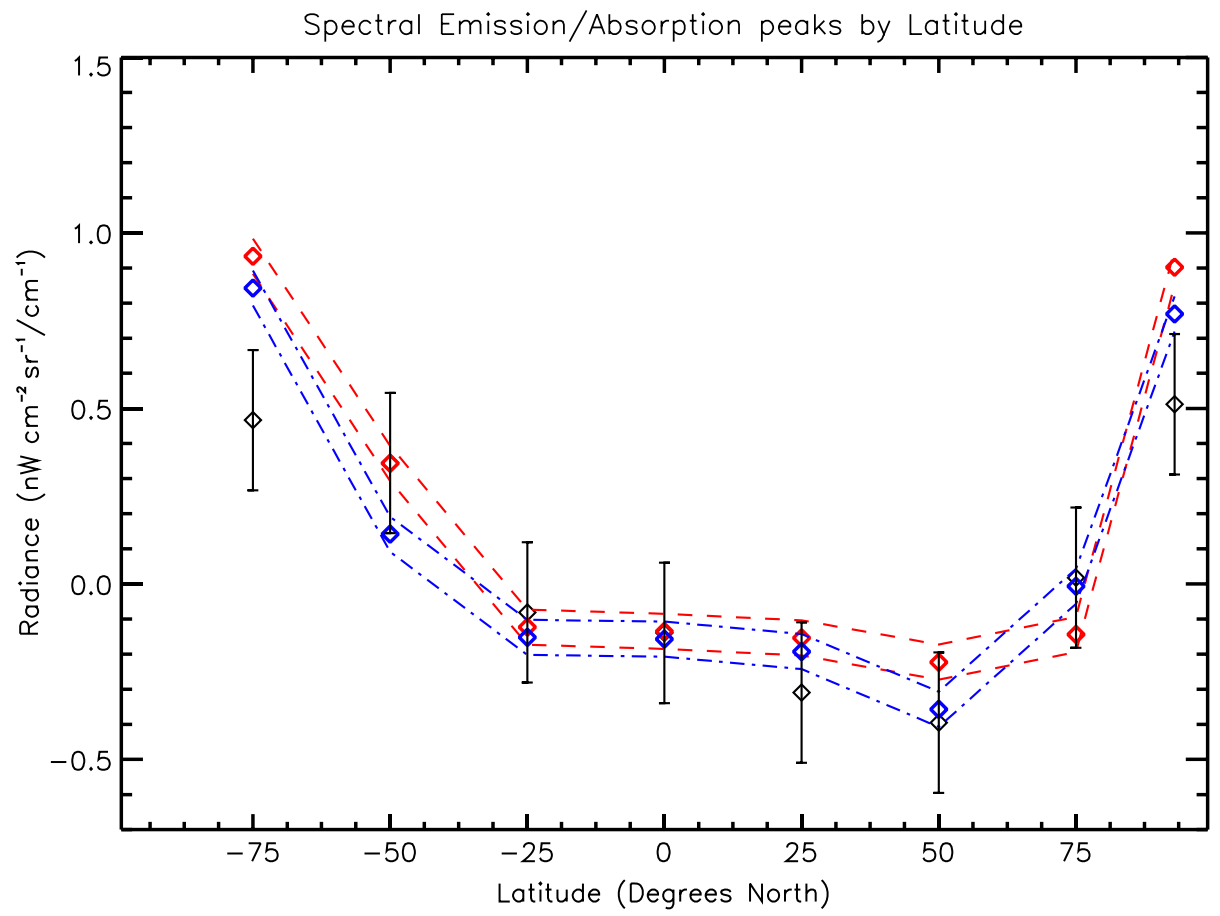

Fig. 9 Line minus continuum peak emissions with 1-sigma RMS error bars (black) compared to NEMESIS models of MCD atmosphere (red) and GEM-Mars (blue) at various latitudes along the subobserver longitude $90^{\circ} \mathrm{E}$. The dashed and dot-dashed lines represent spatial and temporal variation within the MCD and GEM-Mars models, respectively, for the observed latitudes. 


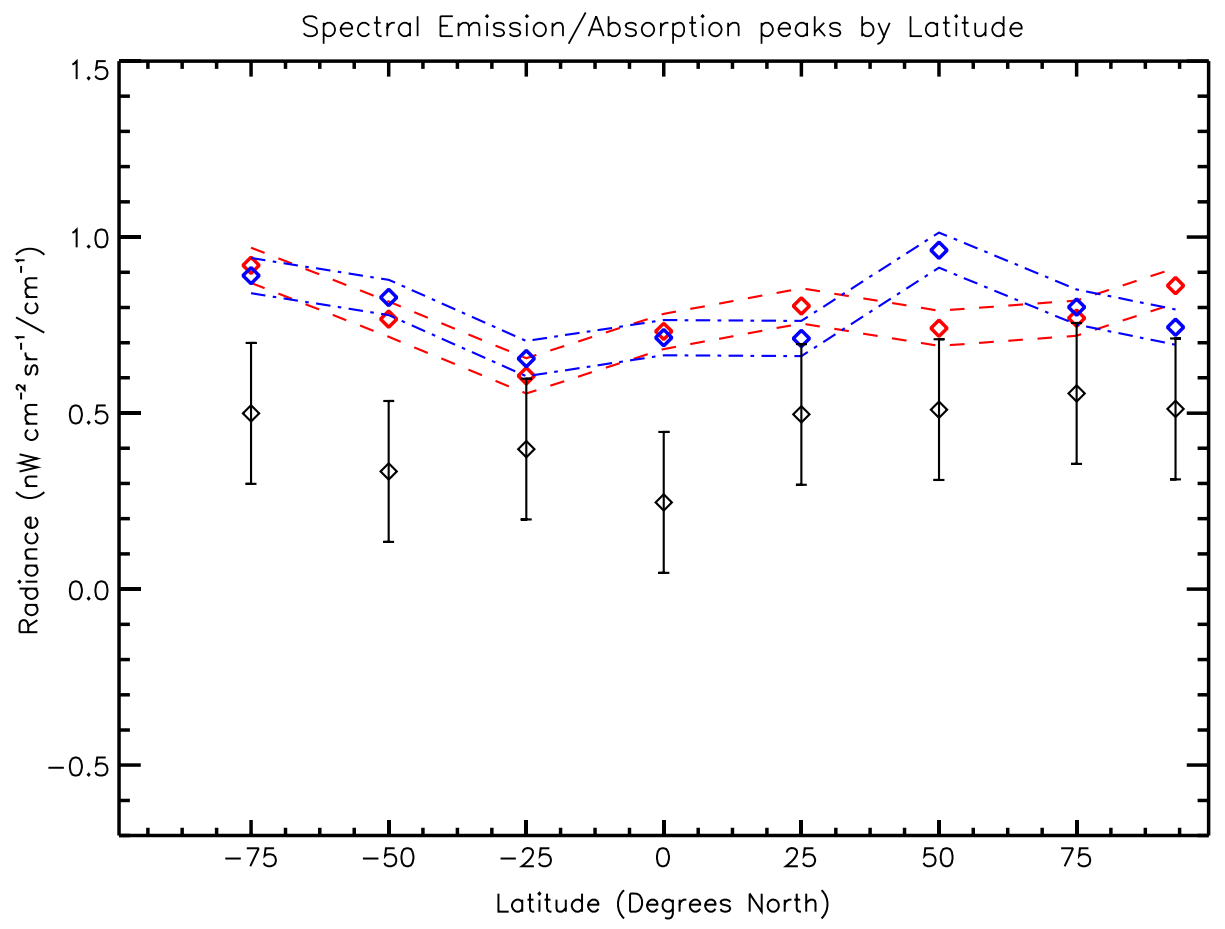

Fig. 10 Observed line peaks (black) compared to NEMESIS models of MCD atmosphere (red) and GEM-Mars (blue) at various latitudes along the eastern limb of the disk of Mars located around $180^{\circ} \mathrm{E}$. As in Fig. 8, the dashed and dot-dashed lines represent spatial and temporal variation within the MCD and GEM-Mars models, respectively, for the observed latitudes.

absorption lines, but without accurate calibration of the emission and absorption lines, there would be no way to retrieve unique information.

\subsection{Retrievals to Assess Plausibility of Limb Emission Features}

First, we confirmed that the data required significant ( $>10 \%)$ scaling of the flux density to match our synthetic continuum after performing standard flux calibration. ${ }^{28} \mathrm{Next}$, to evaluate whether or not the data we were seeing were within the realm of physical possibility, we attempted to retrieve atmospheric properties from these observations.

\subsection{Surface Temperature and Atmospheric Temperature Profile}

To fit the increased line-to-continuum ratio, our retrieved profile required a surface temperature at least $50 \mathrm{~K}$ higher than the models predicted. An example can be shown in Fig. 11, depicting the retrieved temperature profile and spectrum for Mars' northern pole. For the retrieval in Fig. 11, the CO abundance was fixed to the profile modeled by MCD [the red line in Fig. 12(a)].

\subsection{Atmospheric CO Profile}

Adjusting the $\mathrm{CO}$ vertical profile required a factor of at least $3 \times$ to $5 \times$ difference to fit the increased line-to-continuum ratio. An example can be seen in Fig. 12, depicting the retrieved $\mathrm{CO}$ profile and spectrum for Mars' northern pole. The retrieval in Fig. 12 used a fixed temperature profile, which was pulled from MCD [the red line in Fig. 11(a)].

Neither adjusting temperature nor $\mathrm{CO}$ abundance was capable of fitting our spectrum to a physically plausible atmosphere. Without reliable continuum levels or line to continuum ratio, our atmospheric state parameters become degenerate. To further validate our findings, we verified that the telescope array was properly aligned; extracting spectra on points off the expected limb coordinates resulted in a reduction in the spectral features we have attributed to limb 


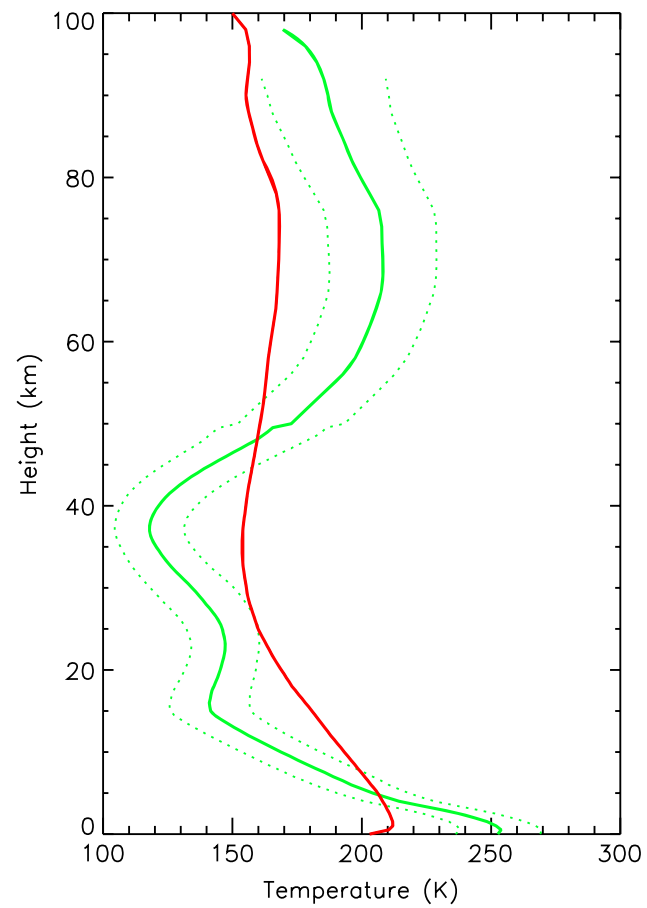

(a)

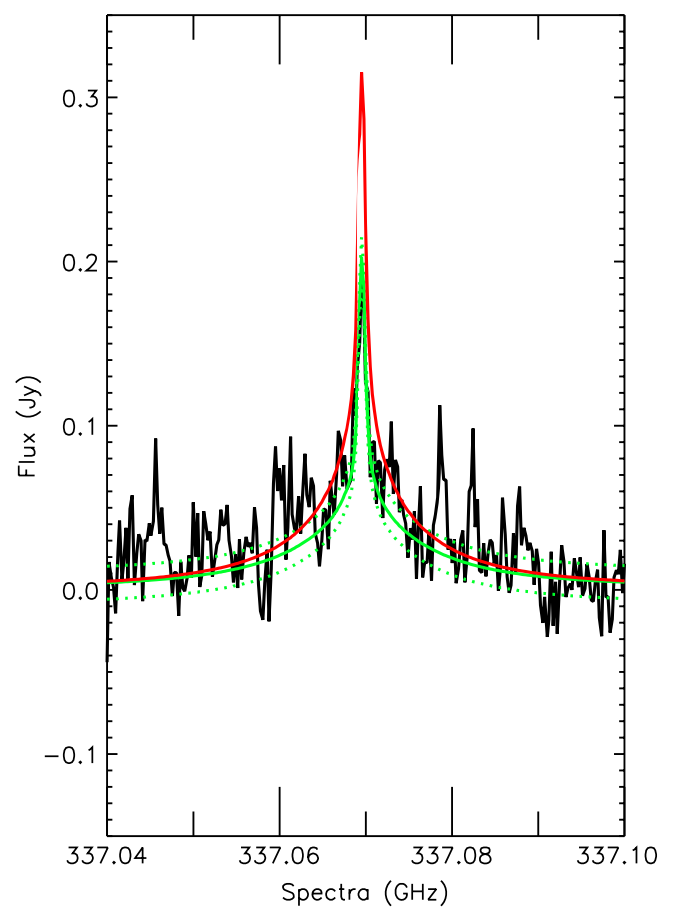

(b)

Fig. 11 (a) Temperature profile modeled by MCD (red) compared to the temperature profile retrieved by NEMESIS (green, with dotted 1-sigma RMS error) that fit the $\mathrm{C}^{17} \mathrm{O}$ transition at the Martian north pole. Across most of the column, the temperature varied from the model by more than $50 \mathrm{~K}$. Finding such deviations from expectation in the lower atmosphere of Mars is dubious. ${ }^{29}$ It is more likely that this retrieval is attempting to fit a nonphysical emission line that has lost flux due to systematic errors within the observation. (b) Continuum subtracted $\mathrm{C}^{17} \mathrm{O}$ transition at the Martian north pole as viewed by ALMA (black) compared with the forward model (red) and best fit retrieval (green, with dotted 1-sigma RMS error) spectra from NEMESIS.

brightening. While it is theoretically possible that the observation was not exactly on target, the analytical degeneracies and lack of reliable continuum flux make perfect validation impossible.

\section{Summary and Conclusions}

The findings in Sec. 4 confirm that this dataset's emission features as calibrated seen in Mars's limb are physically unlikely; it is more likely that the observation's systematic errors affected not just the continuum but the spectral line shapes as well.

Using ground-based radio telescopes to observe spatial distributions of gases on Mars might soon provide the Martian climate community with a new tool with which to validate and refine the currently existing models. However, care must be taken with high-resolution interferometers to account for the maximum recoverable scale and to preserve continuum flux, as to avoid the analytical degeneracies encountered in our work. If properly carried out, ground-based observations could provide complementary data to help fill in any gaps in datasets provided by spacecraft in orbit around Mars.

This work serves to inform future observations made by ALMA so that they might eventually corroborate spatially resolved predictions from global climate models such as MCD or GEM Mars. To properly accommodate the large angular scale of Mars while observing with ALMA, we recommend utilizing the ACA, as at $345 \mathrm{GHz}$ it has an angular resolution of 3.63", with a maximum recoverable scale of 19.3". The ACA is a collection of short-baseline, 7-m dishes that would help fill in continuum flux from Mars and would allow for the proper calibration of highresolution, long-baseline data. Without accurate continuum flux, there is no possible way to verify the integrity of the high-resolution data and retrieve the atmospheric patterns therein. It is paramount that future ALMA Mars observations take this into account. 
Parks et al.: Observations of Mars with ALMA: potential for future constraints of global circulation models
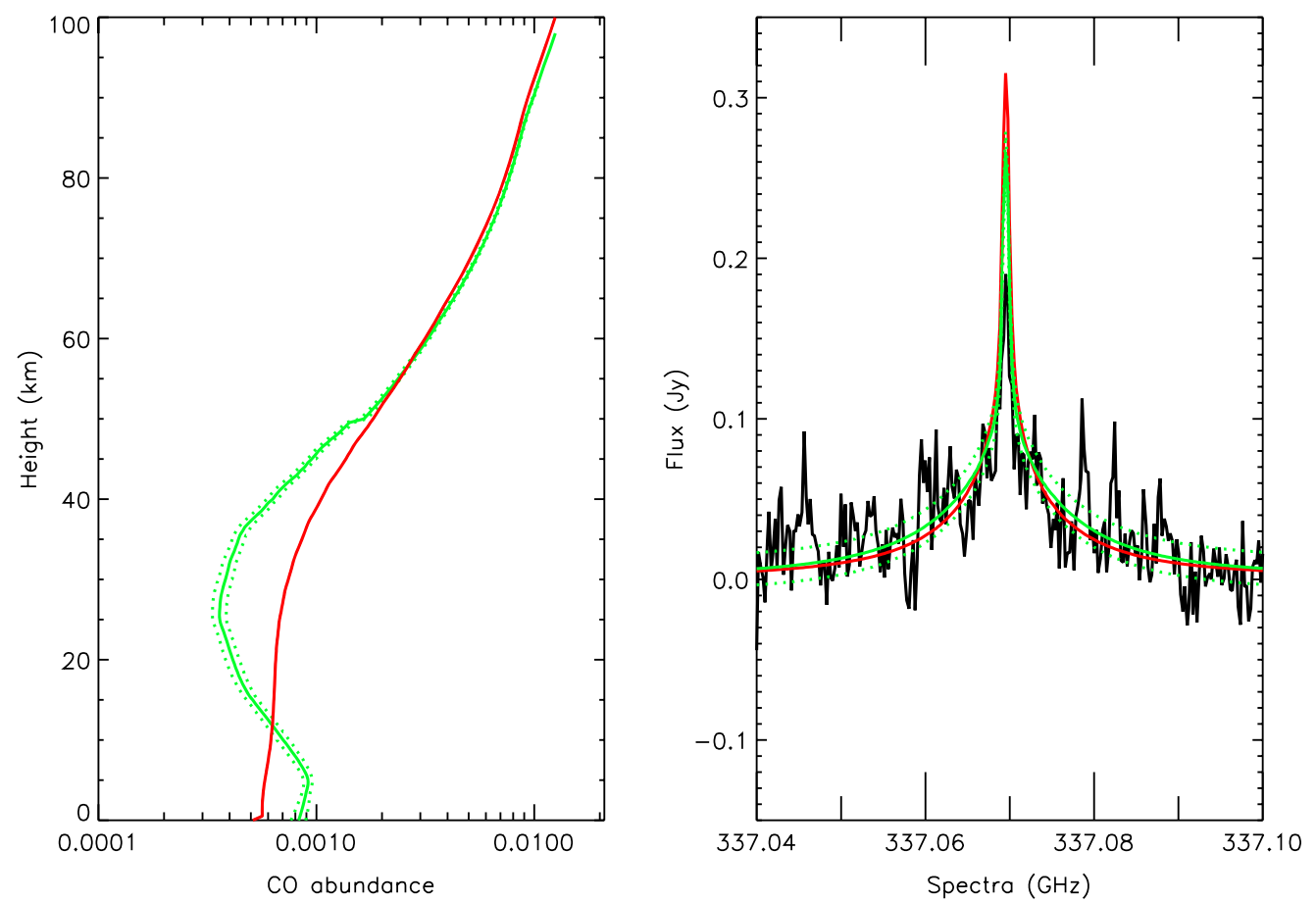

Fig. 12 (a) CO abundance profile modeled by MCD (red) compared to the abundance profile retrieved by NEMESIS (solid green, with dotted green error bounds) that attempted to fit the observed spectrum (black solid line, right-hand side) as in Fig. 11. Across most of the column, the $\mathrm{CO}$ abundance varied from the model by more than $300 \%$. (b) Even with the retrieval's large deviation from the modeled expectation (red), the spectral line peak was not within the retrieved error (green dotted line) of the retrieved spectrum (green solid line). As in Fig. 9, we suspect that this is not realistic and instead likely captures systematic error within our observation.

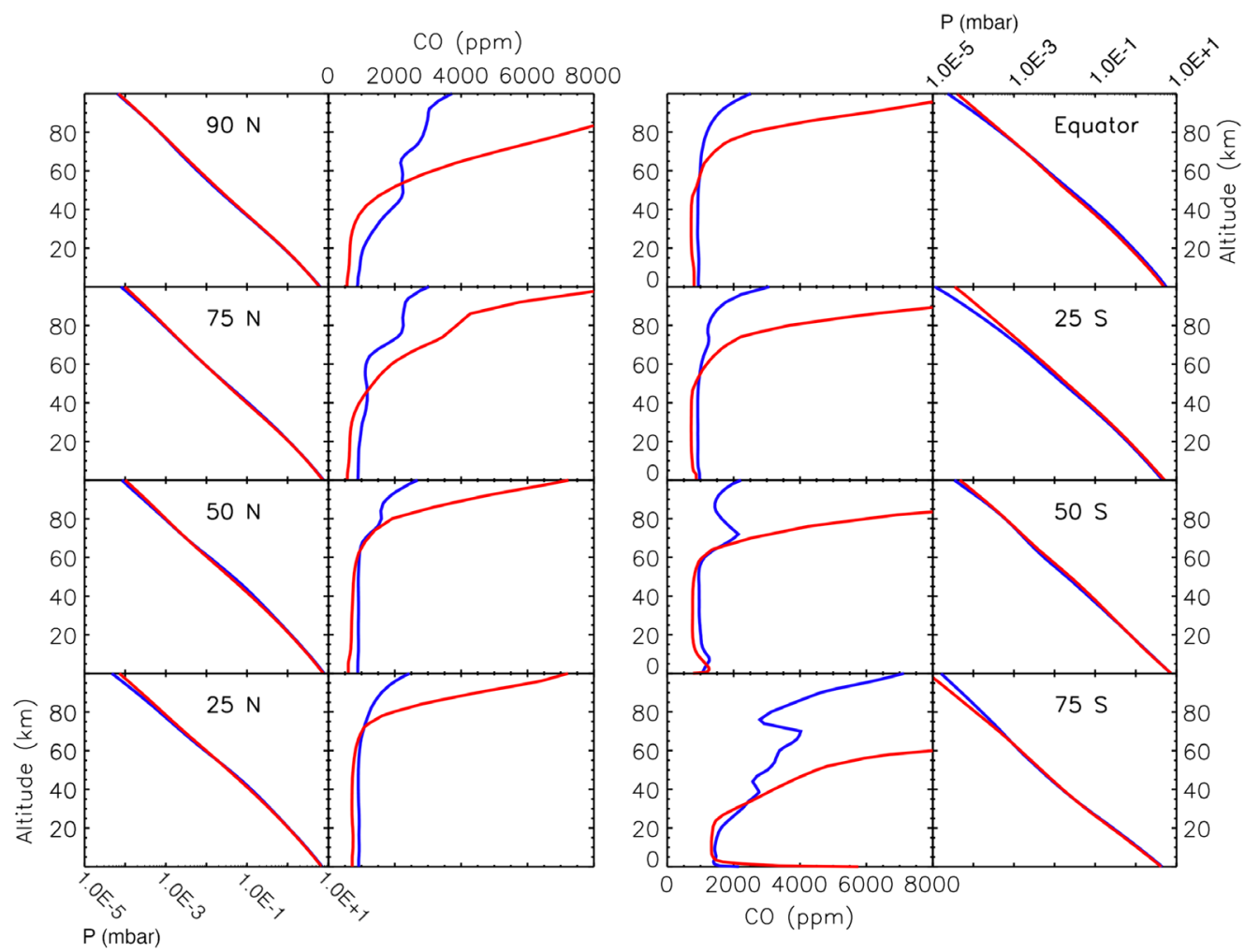

Fig. 13 Shown here are CO VMR profiles and pressure profiles taken from both MCD (red) and GEM-Mars (blue) for the date of our observation. 
Parks et al:: Observations of Mars with ALMA: potential for future constraints of global circulation models

\section{Appendix: CO Abundance and Pressure Profile to Supplement Figs. 7 and 8}

The complete set of reference volume mixing ratio (VMR) and pressure profiles for the latitudes sampled can be seen in Fig. 13. These atmospheric profiles were used to generate the model spectra in figures Star (1) and Star (2), along with the temperature.

\section{Acknowledgments}

M.C.P., C.A.N., A.E.T., \& G.L.V. received funding from NASA Goddard Spaceflight Center's Fundamental Laboratory Research (FLaRe) Program. This paper makes use of the following ALMA data: ADS/JAO.ALMA\#2015.1.01269.S. ALMA is a partnership of ESO (representing its member states), NSF (USA) and NINS (Japan), together with NRC (Canada), MOST and ASIAA (Taiwan), and KASI (Republic of Korea), in cooperation with the Republic of Chile. The Joint ALMA Observatory is operated by ESO, AUI/NRAO, and NAOJ. The National Radio Astronomy Observatory is a facility of the National Science Foundation operated under cooperative agreement by Associated Universities, Inc.

\section{References}

1. M. B. McElroy, T. Y. Kong, and Y. L. Yung, "Photochemistry and evolution of Mars' atmosphere: a viking perspective," J. Geophys. Res. (1896-1977) 82, 4379-4388 (1977).

2. E. Lellouch, G. Paubert, and T. Encrenaz, "Mapping of CO millimeter-wave lines in Mars' atmosphere: the spatial variability of carbon monoxide on Mars," Planet. Sp. Sci. 39, 219-224 (1991).

3. M. D. Smith et al., "Compact reconnaissance imaging spectrometer observations of water vapor and carbon monoxide," J. Geophys. Res.: Planets 114(E2) (2009).

4. M. D. Smith et al., "The climatology of carbon monoxide and water vapor on Mars as observed by CRISM and modeled by the GEM-Mars general circulation model," Icarus 301, 117-131 (2018).

5. J. Rosenqvist et al., "The vertical distribution of carbon monoxide on Mars from the ISMPhobos experiment," Astron. Astrophys. 231, L29-L32 (1990).

6. A. S. Khayat et al., "A search for $\mathrm{SO}_{2}, \mathrm{H}_{2} \mathrm{~S}$ and $\mathrm{SO}$ above Tharsis and Syrtis volcanic districts on Mars using ground-based high-resolution submillimeter spectroscopy," Icarus 253, 130-141 (2015).

7. A. S. Khayat et al., "A deep search for the release of volcanic gases on Mars using groundbased high-resolution infrared and submillimeter spectroscopy: sensitive upper limits for OCS and $\mathrm{SO}_{2}$," Icarus 296, 1-14 (2017).

8. G. L. Villanueva, "The high resolution spectrometer for SOFIA-GREAT instrumentation, atmospheric modeling and observations," PhD Thesis (2004).

9. "ISM observation sessions," https://www.ias.u-psud.fr/ism/DRV_DATA/HTML/ISM/ COUVISM.HTM (2001).

10. R. T. Clancy and B. J. Sandor, "Mesospheric winds and temperatures from JCMT submillimeter co line observations during the 2003 and 2005 mars oppositions," in Second Mars Atmos. Modell. and Obs. Workshop, Granada (2006).

11. R. Moreno et al., "Wind measurements in Mars' middle atmosphere: IRAM Plateau de Bure interferometric CO observations," Icarus 201, 549-563 (2009).

12. J. P. McMullin et al., "CASA architecture and applications," in Astronomical Data Analysis Software and Systems XVI ASP Conf. Series, Vol. 376, pp. 127 (2007).

13. F. Forget et al., "Improved general circulation models of the Martian atmosphere from the surface to above $80 \mathrm{~km}, "$ J. Geophys. Res.: Planets 104, 24155-24175 (1999).

14. E. Millour et al., "The mars climate database (Version 5.3)," in Sci. Workshop: "From Mars Express to ExoMars" (2018).

15. P. G. J. Irwin et al., "The NEMESIS planetary atmosphere radiative transfer and retrieval tool," J. Quant. Spectrosc. Radiat. Transfer 109, 1136-1150 (2008). 
Parks et al.: Observations of Mars with ALMA: potential for future constraints of global circulation models

16. M. A. Cordiner et al., "Ethyl cyanide on titan: spectroscopic detection and mapping using ALMA," Astrophys. J. 800, L14 (2015).

17. J. Serigano et al., "Isotopic ratios of carbon and oxygen in Titan's CO using ALMA," Astrophys. J. 821, L8 (2016).

18. M. Y. Palmer et al., "ALMA detection and astrobiological potential of vinyl cyanide on Titan," Sci. Adv. 3, e1700022 (2017).

19. N. A. Teanby et al., "Constraints on Titan's middle atmosphere ammonia abundance from Herschel/SPIRE sub-millimetre spectra," Planet. Sp. Sci. 75, 136-147 (2013).

20. I. E. Gordon et al., "The HITRAN2016 molecular spectroscopic database," J. Quant. Spectrosc. Radiat. Transfer 203, 3 (2017).

21. L. S. Rothman et al., "The HITRAN2012 molecular spectroscopic database," J. Quant. Spectrosc. Radiat. Transfer 130, 4 (2013).

22. A. E. Thelen et al., "Spatial variations in Titan's atmospheric temperature: ALMA and Cassini comparisons from 2012 to 2015," Icarus 307, 380-390 (2018).

23. S. P. Belov, M. I. Tret'iakov, and R. D. Suenram, "Improved laboratory rest frequency measurements and pressure shift and broadening parameters for the $\mathbf{J}=21$ and $\mathbf{J}=32$ rotational transitions of CO," Astrophys. J. 393, 848 (1992).

24. A. E. Thelen et al., "Measurement of $\mathrm{CH} 3 \mathrm{D}$ on Titan at submillimeter wavelengths," Astron. J. 157, 219 (2019).

25. "NASA planetary spectrum generator," https://psg.gsfc.nasa.gov/.

26. G. L. Villanueva et al., "Planetary spectrum generator: an accurate online radiative transfer suite for atmospheres, comets, small bodies and exoplanets," J. Quant. Spectrosc. Radiat. Transfer 217, 86-104 (2018).

27. L. Neary and F. Daerden, "The GEM-Mars general circulation model for Mars: description and evaluation," Icarus 300, 458-476 (2018).

28. E. Fomalont et al., "The calibration of ALMA using radio sources," Telesc. Instrum., 155, 19-22 (2014).

29. M. D. Smith et al., The Atmospheric and Climate of Mars, R. M. Haberle et al., Ed., Cambridge University Press (2017).

Maxwell C. Parks is a graduate student at the University of California Los Angeles. He received his BS degree in physics, his BS degree in astronomy, and his BA degree in humanities from the University of Texas at Austin in 2017. This is his first journal paper. His current research interests include global atmospheric models of Mars, both past and present.

Conor A. Nixon is a planetary scientist at NASA's Goddard Space Flight Center, specializing in the study of planetary atmospheres. He has worked on the Galileo and Cassini missions and ground-based observing with IRTF and ALMA. He is the lead for Titan science in the JWST GTO program.

Geronimo L. Villanueva is a planetary scientist at NASA-GSFC who specializes in the search for organic molecules on Mars and on icy bodies. He is the principal investigator (PI) of the Planetary Spectrum Generator (PSG), co-PI of the Comet Interceptor Mission, co-investigator of the ExoMars/TGO mission, and leader for Mars and Ocean Worlds studies (GTO) for JWST. He has a long experience employing ground-based observatories, including Keck, NASA-IRTF, and ALMA.

Michael D. Smith is a planetary scientist in the Planetary Systems Lab of the NASA Goddard Space Flight Center. His research interests include the meteorology and dynamics of planetary atmospheres, radiative transfer, and remote sensing techniques. He has been an active participant on the science teams of numerous orbital and landed spacecraft missions to Mars, including Mars Reconnaissance Orbiter, Mars Science Laboratory rover, ExoMars Trace Gas Orbiter, Mars Perseverance rover, and the Emirates Mars Mission.

Alain S. J. Khayat is an assistant research scientist at NASA Goddard Space Flight Center. His research focuses on the remote sensing of the atmosphere of Mars, by developing radiative transfer models from near infrared to submillimeter wavelengths. This includes ground-based 
(NASA IRTF, Keck, JCMT, SMA, CSO), spacecraft-based (MRO/CRISM, TGO/NOMAD), and rover-based (Mars Science Laboratory) observations. Analyses include the study of the water cycle on Mars, atmospheric D/H mapping, and trace gases.

Alexander E. Thelen received his BS degree in physics from the University of California, Santa Cruz, in 2012, and his PhD in astronomy from New Mexico State University in 2018. He is currently a NASA Astrobiology Postdoctoral Program fellow at NASA Goddard Space Flight Center in the Planetary Systems Laboratory. His research interests include remote sensing of planetary atmospheres at (sub)millimeter wavelengths, radiative transfer modeling, and mission concept development.

Eric Villard is an astronomer working for the European Southern Observatory (ESO) in Garching. He received his PhD in planetary science in 2008 on the study of the atmosphere of Venus with the SPICAV instrument onboard Venus Express. He then worked at the Joint ALMA Observatory in Chile, on the commissioning and science verification of the observatory. He is now working on the development of advanced data products for the ESO observatories.

Steven B. Charnley is a physical scientist at NASA Goddard Space Flight Center. His research involves theoretical and observational studies of molecular astrophysics and astrochemistry in a variety of astronomical and solar system environments. These include interstellar clouds, regions of star formation, evolved stars, comets, planets, and moons.

Patrick G. J. Irwin is a professor of planetary physics at the University of Oxford and has more than 30 years' experience in remote sensing of planetary atmospheres from satellite and groundbased observations. He is the originator and lead developer of the NEMESIS radiative transfer and retrieval code and leads an active programme of ground-based spectroscopic observations (and interpretation) of solar system planetary atmospheres. 